\title{
Subtracting compact binary foreground sources to reveal primordial gravitational-wave backgrounds
}

\author{
Surabhi Sachdev@ , $^{1, *}$ Tania Regimbau, ${ }^{2, \dagger}$ and B. S. Sathyaprakash ${ }^{1,3,4, \ddagger}$ \\ ${ }^{1}$ Institute for Gravitation and the Cosmos, Physics Department, Pennsylvania State University, \\ University Park, Pennsylvania 16802, USA \\ ${ }^{2}$ LAPP, Universit'e Grenoble Alpes, USMB, CNRS/IN2P3, F-74000 Annecy, France \\ ${ }^{3}$ Department of Astronomy and Astrophysics, Pennsylvania State University, \\ University Park, Pennsylvania 16802, USA \\ ${ }^{4}$ School of Physics and Astronomy, Cardiff University, Cardiff, CF24 3AA, United Kingdom
}

(Received 14 May 2020; accepted 9 June 2020; published 16 July 2020)

\begin{abstract}
Detection of primordial gravitational-wave backgrounds generated during the early Universe phase transitions is a key science goal for future ground-based detectors. The rate of compact binary mergers is so large that their cosmological population produces a confusion background that could masquerade the detection of potential primordial stochastic backgrounds. In this paper, we study the ability of current and future detectors to resolve the confusion background to reveal interesting primordial backgrounds. The current detector network of LIGO and Virgo and the upcoming KAGRA and LIGO-India will not be able to resolve the cosmological compact binary source population, and its sensitivity to stochastic background will be limited by the confusion background of these sources. We find that a network of three (and five) third generation $(3 \mathrm{G})$ detectors of Cosmic Explorer and Einstein Telescope will resolve the confusion background produced by binary black holes leaving only about $1.3 \%$ (respectively, $0.075 \%$ ) unresolved; in contrast, as many as $25 \%$ (respectively, $7.7 \%$ ) of binary neutron star sources remain unresolved. Consequently, the binary black hole population will likely not limit observation of primordial backgrounds, but the binary neutron star population will limit the sensitivity of $3 \mathrm{G}$ detectors to $\Omega_{\mathrm{GW}} \sim 10^{-11}$ at $10 \mathrm{~Hz}$ (respectively, $\Omega_{\mathrm{GW}} \sim 3 \times 10^{-12}$ ).
\end{abstract}

DOI: $10.1103 /$ PhysRevD.102.024051

\section{INTRODUCTION}

With the continued detections of gravitational waves from binary black hole mergers [1-6] and binary neutron star inspirals [7,8], the LIGO Scientific and Virgo Collaborations have kept up to their promise of taking us into an era of gravitational-wave astronomy. In addition to these loud and nearby sources that are seen as isolated transient events, there is a population of weak, unresolved sources at higher redshifts [9-13]. The superposition of these sources is expected to be the main contributor to the astrophysical stochastic background which may be detectable in the next few years as the Advanced LIGO [14] and Virgo detectors [15] reach their design sensitivity and accumulate more data $[16,17]$. Assuming the most probable rate for compact binary

\footnotetext{
*szs1416@psu.edu

tania.regimbau@lapp.in2p3.fr

"bss25@psu.edu
}

Published by the American Physical Society under the terms of the Creative Commons Attribution 4.0 International license. Further distribution of this work must maintain attribution to the author(s) and the published article's title, journal citation, and DOI. mergers at the time $\left(103_{-63}^{+110} \mathrm{Gpc}^{-3} \mathrm{yr}^{-1}\right.$ [3] for binary black hole (BBH) and $1540_{-1220}^{+3200} \mathrm{Gpc}^{-3} \mathrm{yr}^{-1}$ [7] for binary neutron star (BNS)), it has been shown that the total background may be detectable with a signal-to-noise-ratio of 3 after 40 months of total observation time, based on the expected timeline for Advanced LIGO and Virgo to reach their design sensitivity [17]. The astrophysical background potentially contains a wealth of information about the history and evolution of a population of point sources, but it is a confusion noise background that obscures the observation of the primordial gravitational-wave background produced in the very early stages of the Universe. Proposed theoretical cosmological models include the amplification of vacuum fluctuations during inflation [18-20], pre-bigbang models [21-23], cosmic (super) strings [24-27], or phase transitions [28-30]. For a comprehensive discussion of cosmological gravitational-wave backgrounds, we refer the reader to reviews by Maggiore [31] and Binétruy et al. [32].

Detection of the primordial gravitational-wave background would create a unique window on the earliest moments of the Universe, up to the limits of the Planck era, and on the physical laws that apply at the highest energy 
scales. Needless to say that such a detection would have a profound impact on our understanding of the evolution of the Universe.

In addition to the astrophysical background from unresolved compact binary mergers, a contribution is expected to result from the superposition of several other unresolved sources [33], such as cosmic (super) strings [25], core collapse supernovae to neutron stars or black holes [34-37], rotating neutron stars [38,39] including magnetars [40-43], phase transitions [44], or initial instabilities in young neutron stars [45-47].

The current detector network of LIGO and Virgo and the upcoming KAGRA and LIGO-India will not be able to resolve the cosmological compact binary source population, and its sensitivity to stochastic background will be limited by the confusion background of these sources [48]. With the increased sensitivity of the third generation gravitationalwave detectors, such as the Einstein Telescope (ET) [49] and the Cosmic Explorer (CE) [50], it will be possible to detect and resolve almost all of the binary black hole mergers, even the ones at high redshifts. In this work, we explore the possibility of probing the cosmological gravitational-wave background with the third generation detectors, after removing the astrophysical background from compact binary mergers from the data. This work is an extension to [48], where the authors have shown the level at which we can expect amplitude of background from unresolved, subthreshold signals from compact binary coalescences (CBC) using different detector networks. We extend the previous study to also provide an estimate of errors we introduce while subtracting the signals above threshold for the most optimistic network of detectors considered by [48]. The idea of subtracting foreground signals to extract stochastic backgrounds was already explored [51] in the context of the big bang observer [52], including a noise projection method that could reduce errors due to imperfect subtraction [53].

Data from gravitational-wave detectors are dominated by environmental and instrumental backgrounds. Consequently, it is not possible to identify even deterministic signals without sophisticated data processing such as matched filtering [54]. Stochastic backgrounds cannot be reliably detected in a single detector - they are found by cross-correlating the data from a pair of detectors. Indeed, the stochastic background present in one of the detectors acts as a matched filter for the data in the other detector [55-57]. Unfortunately, this means that any common noise in a pair of detectors could masquerade as stochastic background [58]. If detectors are geographically well separated, then the risk of common noise of terrestrial origin is greatly reduced. Additionally, certain backgrounds of terrestrial origin could be measured and subtracted [59]. Even in the absence of any terrestrial background, a pair of detectors would see the same astrophysical background, which would show up as correlated "noise," although detectors might be geographically well separated. As a result, the only possible way to improve the sensitivity of a detector network to primordial backgrounds is to subtract foreground astrophysical signals.

The rest of the paper is organized as follows. In Sec. II, we describe the basic method that we use to calculate the gravitational-wave spectrum from the error introduced by imperfect subtraction of $\mathrm{CBC}$ signals. In Sec. III, we describe the framework used to estimate the deviations of the estimated parameters of the CBC sources from their true values. We discuss the simulation of a population of binaries in Sec. IV, discuss the result of the imperfect subtraction of such signals in Sec. V, and we discuss our results in Sec. VI.

\section{METHOD}

The energy-density spectrum in gravitational waves is described by the dimensionless quantity [57],

$$
\Omega_{\mathrm{GW}}(f)=\frac{f}{\rho_{c}} \frac{d \rho_{\mathrm{GW}}}{d f},
$$

where $d \rho_{\mathrm{GW}}$ is the energy density in the frequency interval $f$ to $f+d f, \rho_{c}=3 H_{0}^{2} c^{2} / 8 \pi G$ is the closure energy density, and $H_{0}$ is the Hubble constant equal to $67.8 \pm 0.9 \mathrm{~km} / \mathrm{c} / \mathrm{Mpc}[60]$.

The gravitational-wave energy spectrum density can be written as a sum of contribution from the astrophysical and cosmological energy densities,

$$
\Omega_{\mathrm{GW}}=\Omega_{\mathrm{astro}}+\Omega_{\text {cosmo }} .
$$

Taking the contribution of the compact binary coalescences out of the astrophysical background, and writing it explicitly, we have

$$
\Omega_{\mathrm{GW}}=\Omega_{\mathrm{astro}, \mathrm{r}}+\Omega_{\mathrm{cosmo}}+\Omega_{\mathrm{cbc}} .
$$

Here $\Omega_{\mathrm{astro,r}}$ is the remaining astrophysical background after taking out the contribution from the CBC sources.

When estimating the parameters of a binary source, by using Monte Carlo methods, or nested sampling, we invariably end up with parameters that deviate from the true values because of the noise in the detector. Therefore, when we subtract the recovered $\mathrm{CBC}$ signals from the data, we introduce an additional background due to the error in subtraction, $\Omega_{\text {error }}$,

$\Omega_{\mathrm{GW}}=\Omega_{\mathrm{cbc} \text {, rec }}+\Omega_{\mathrm{error}}+\Omega_{\mathrm{cbc}, \text { unres }}+\Omega_{\mathrm{cosmo}}+\Omega_{\mathrm{astro}, \mathrm{r}}$,

where $\Omega_{\mathrm{cbc} \text {, rec }}$ is the background from the recovered CBC sources that we can subtract from our data, $\Omega_{\text {error }}$ is the background because of the error introduced from such a subtraction, $\Omega_{\mathrm{cbc} \text {, unres }}$ is the background from the unresolved CBC sources which are not detected as foreground events. Let us assume that we have an experiment where we 
have detected a list of $\mathrm{CBC}$ sources and subtracted them from the data. Now, we are left with the gravitational-wave backgrounds, $\Omega_{\mathrm{error}}, \Omega_{\mathrm{cbc} \text {, unres, }}$, on top of the cosmological and astrophysical (from sources other than the CBCs) backgrounds. We want to answer the question of whether the cosmological or astrophysical backgrounds from sources other than $\mathrm{CBCs}$ can stand above the residual background after removal of the CBC sources. That is,

$$
\Omega_{\text {error }}+\Omega_{\text {cbc, unres }} \stackrel{?}{\lessgtr} \Omega_{\text {cosmo }} \stackrel{?}{\lessgtr} \Omega_{\text {astro, } \mathrm{r}} .
$$

In order for us to be able to detect the gravitational-wave background from cosmological sources or that from different astrophysical sources, we would need $\Omega_{\text {residual }}=$ $\Omega_{\text {error }}+\Omega_{\text {cbc, unres }}$ to lie below these.

The gravitational-wave energy density from a population of compact binary sources is given by [48]

$$
\Omega_{\mathrm{cbc}}=\frac{1}{\rho_{c} c} f F(f),
$$

where $F(f)$ is the total flux, sum of individual contributions

$$
F(f)=T^{-1} \frac{\pi c^{3}}{2 G} f^{2} \sum_{k=1}^{N}\left(\tilde{h}_{+, k}^{2}(f)+\tilde{h}_{\times, k}^{2}(f)\right),
$$

where $N$ is the number of sources in the Monte Carlo sample, and $T^{-1}$ assures that flux has the correct dimension, $T$ being the total time of the data sample. $\tilde{h}_{+, k}(f)$ and $\tilde{h}_{\times, k}(f)$ are the Fourier domain waveforms for the two polarizations, and the index $k$ runs over all the sources. We calculate $\Omega_{\text {error }}$ as

$$
\Omega_{\mathrm{error}}=\frac{1}{\rho_{c} c} f F_{\text {error }}(f),
$$

where

$$
\begin{aligned}
F_{\text {error }}(f)= & T^{-1} \frac{\pi c^{3}}{2 G} f^{2} \sum_{k=1}^{N}\left(\left(\tilde{h}_{+, k}^{\text {true }}(f)-\tilde{h}_{+, k}^{\text {recovered }}(f)\right)^{2}\right. \\
& \left.+\left(\tilde{h}_{\times, k}^{\text {true }}(f)-\tilde{h}_{\times, k}^{\text {recovered }}(f)\right)^{2}\right) .
\end{aligned}
$$

To get an estimate of $\Omega_{\text {error }}$, we need to estimate the quantities, $\tilde{h}_{+, k}^{\text {recovered }}(f)$ and $\tilde{h}_{\times, k}^{\text {recovered }}(f)$.

\section{ESTIMATING THE DEVIATION FROM TRUE VALUE OF THE MEASURED SOURCE PARAMETERS}

Ideally, we want the full Bayesian posteriors to estimate the deviation from the true value of parameters. However, at present, it is unfeasible to compute the full posterior probability distribution functions of all 15 binary parameters for the hundreds of thousands of sources that we simulate up to a redshift of 10 in the following section. The Fisher matrix provides a computationally inexpensive method to estimate the errors in the case when the posteriors are Gaussian, which is, unfortunately, not true in general. Nevertheless, for the purpose of building a proof-of-principle concept, the Fisher matrix method is adequate and the only practical approach to obtain the magnitude of errors in the estimation of parameters. To this end, we follow the framework described in [61] and calculate the errors in estimating the parameters of the compact binary system using the Fisher matrix method.

According to the post-Newtonian expansion formalism [62], the gravitational-wave strain from a compact binary coalescence in frequency domain is given by

$$
\tilde{h}(f)=\mathcal{A} f^{-7 / 6} e^{i \Psi(f)},
$$

where $A$ is the amplitude of the waveform, and $\Psi(f)$ is the phase given by

$$
\Psi(f)=2 \pi f t_{c}-\phi_{c}-\frac{\pi}{4}+\frac{3}{128 \eta \nu^{5}} \sum_{k=0}^{N} \alpha_{k} \nu^{k} .
$$

Here $t_{c}$ is the time of coalescence, $\phi_{c}$ is the coalescence phase, $\nu=(\pi M f)^{1 / 3}, M$ is the total mass $\left(M=m_{1}+m_{2}\right)$, $\eta$ is the symmetric mass ratio $\left(\eta=m_{1} m_{2} / M^{2}\right)$ of the system, and the $\alpha_{k}$ terms are known as the postNewtonian (PN) coefficients. In this work, we restrict ourselves to 0-PN approximation (or the Newtonian approximation, $k=0$ ), which will be justified below. For the Fisher matrix study, we choose a set of independent parameters $\vec{\theta}$ for describing the gravitational waveform,

$$
\vec{\theta}=\left(f_{0} t_{c}, \phi_{c}, \ln \mathcal{M}\right),
$$

where $f_{0}$ is a reference frequency needed to keep the parameters for the Fisher matrix dimensionless. $\mathcal{M}$ is the dimensionless chirp mass and is defined as $\mathcal{M}=$ $\eta^{3 / 5} M / M_{\odot}$.

Writing the phase of the waveform in terms of these parameters, we have

$\Psi(f)=2 \pi \frac{f}{f_{0}}\left(f_{0} t_{c}\right)-\phi_{c}-\frac{\pi}{4}+\frac{3}{128}(\pi \mathcal{M} f)^{-5 / 3}$,

or equivalently,

$\Psi(f ; \vec{\theta})=2 \pi \frac{f}{f_{0}} \theta_{1}-\theta_{2}-\frac{\pi}{4}+\frac{3}{128}\left(\frac{\pi e^{\theta_{3}} f G M_{\odot}}{c^{3}}\right)^{-5 / 3}$.

In going from Eq. (13) to Eq. (14), we have truncated the expansion at $\alpha_{0}$ term, plugged in the value $\alpha_{0}=1$, and we have introduced the Newton's constant $G$, the speed of 
light $c$, and solar mass $M_{\odot}$, explicitly to keep all quantities in the Eq. (13) dimensionless, and defined masses in solar mass units.

The Fisher matrix elements are given by

$\Gamma_{i j}=2 \int_{f_{L}}^{f_{H}} \frac{\tilde{h}_{\theta_{i}}^{*}(f ; \vec{\theta}) \tilde{h}_{\theta_{j}}(f ; \vec{\theta})+\tilde{h}_{\theta_{i}}(f ; \vec{\theta}) \tilde{h}_{\theta_{j}}^{*}(f ; \vec{\theta})}{S_{n}(f)} \mathrm{d} f$,

where

$$
\tilde{h}_{\theta_{i}}(f ; \vec{\theta})=\frac{\partial \tilde{h}(f ; \vec{\theta})}{\partial \theta_{i}}
$$

are the partial derivatives of the waveform with respect to $\theta_{i}$, the parameters of the waveforms, and $S_{n}(f)$ is the single-sided power spectral density of the detector. The partial derivatives of the waveform can be calculated analytically,

$$
\begin{gathered}
\tilde{h}_{\theta_{1}}(f ; \vec{\theta})=\frac{2 \pi f A}{f_{0}} f^{-7 / 6} e^{i(\Psi(f ; \vec{\theta})+\pi / 2)}, \\
\tilde{h}_{\theta_{2}}(f ; \vec{\theta})=A f^{-7 / 6} e^{i(\Psi(f ; \vec{\theta})-\pi / 2)},
\end{gathered}
$$

and

$$
\tilde{h}_{\theta_{3}}(f ; \vec{\theta})=A f^{-7 / 6} e^{i(\Psi(f ; \vec{\theta})-\pi / 2)} \frac{5}{128}\left(\frac{\pi e^{\theta_{3}} f G}{c^{3}}\right)^{-5 / 3} .
$$

The Fisher matrix is then calculated by performing the integration in Eq. (15) numerically. For a network of detectors, the Fisher matrix is the sum of Fisher matrices for individual detectors,

$$
\Gamma_{i j}^{\mathrm{net}}=\sum_{\operatorname{det}} \Gamma_{i j}^{\mathrm{det}}
$$

The variance-covariance matrix, or simply the covariance matrix, defined as the inverse of the Fisher information matrix, is given by

$$
\Sigma_{i j}=\left(\Gamma^{-1}\right)_{i j}
$$

Once we have the covariance matrix, we use a multivariate normal random number generator to generate observed values of the parameters, $\mathbf{P}_{\mathbf{O}}$, based on the multivariate distribution with the mean equal to the true value of the parameters, $\mathbf{P}_{\mathbf{T}}$, and covariance matrix as $\Sigma$. The error in parameter estimation is then given by

$$
\mathbf{R}=\left[\Delta \theta_{1}, \Delta \theta_{2}, \Delta \theta_{3}\right]=\mathbf{P}_{\mathbf{O}}-\mathbf{P}_{\mathbf{T}},
$$

where

$$
\Delta t_{c}=\frac{\Delta \theta_{1}}{f_{0}}, \quad \Delta \phi_{c}=\Delta \theta_{2}, \quad \Delta \mathcal{M}=\mathcal{M} \Delta \theta_{3} .
$$

\section{POPULATION SYNTHESIS FOR MULTIPLE DETECTORS}

We simulate a population of binary black hole and binary neutron star systems up to a redshift of 10 , and then calculate an estimate of $\Omega_{\mathrm{cbc} \text {, rec }}$ and $\Omega_{\text {error }}$ as outlined in Secs. II and III. The list of compact binaries (neutron star binaries or black hole binaries) is generated following a Monte Carlo procedure described in [48,63-65] and using the fiducial model of [17] for the distribution of the parameters (masses, redshift, position on the sky, polarization, and inclination angle of the binary). In particular, we assume a redshift distribution which is derived from the star formation rate (SFR) of [66] and accounts for a delay between the formation of the progenitors and the merger. We further consider the median rates estimated from the first LIGO observation run.

(1) For BBHs, the intrinsic masses $m_{1}, m_{2}$ (in the source frame) are selected from the power-law distribution (Saltpeter initial mass function [67]) considered in $[3,68]$ of the primary (i.e., the larger mass) companion $p\left(m_{1}\right) \propto m_{1}^{-2.35}$ and from a uniform distribution of the secondary companion. In addition, we require that the component masses take values in the range $5-50 M_{\odot}$.

For BNSs, the intrinsic masses $m_{1}, m_{2}$ (in the source frame) are both drawn from a Gaussian distribution centered around $1.33 M_{\odot}$ with a standard deviation of $0.09 M_{\odot}$.

(2) The redshift $z$ is drawn from a probability distribution $p(z)$ given by

$$
p(z)=\frac{R_{z}(z)}{\int_{0}^{10} R_{z}(z) d z},
$$

obtained by normalizing the merger rate of binaries in the observer frame, $R_{z}(z)$ per interval of redshift, over the range $z \in[0,10]$. We choose to cut off the redshift integral at $z_{\max }=10$, since redshifts larger than 5 contribute little to the background [17]. The merger rate in the observer frame is ${ }^{1}$

$$
R_{z}(z)=\frac{R_{m}(z)}{1+z} \frac{d V}{d z}(z)
$$

where $d V / d z$ is the comoving volume element and $R_{m}(z)$ is the rate per comoving volume in the source frame, given by

\footnotetext{
${ }^{1}$ There was an error in Eq. (2) in [48], which we have corrected it here in Eq. (25).
} 


$$
R_{m}(z)=\int_{t_{\min }}^{t_{\max }} \int_{z_{f}=z\left(t_{m}-t_{d}\right)} R_{f}\left(z_{f}\right) p\left(t_{d}\right) d z_{f} d t_{d}
$$

where $R_{f}\left(z_{f}\right)$ is the binary formation rate as a function of the redshift at formation time, $z_{f}=$ $z\left(t_{f}\right)$ is the source redshift at formation, $p\left(t_{d}\right)$ is the distribution of the time delay $t_{d}$ between the formation and merger of the binary, $z=z\left(t_{m}\right)$ is the source redshift at merger. The integration in Eq. (26) over $z_{f}$ is performed for all the redshifts corresponding to $t_{f}$ such that $t_{m}=t_{f}+t_{d}$.

We consider a time delay distribution $p\left(t_{d}\right) \propto$ $1 / t_{d}$, for $t_{\min }<t_{d}<t_{\max }$. For BNS, we set $t_{\min }=$ 20 Myr [17,69], whereas for $\mathrm{BBH}$, we set $t_{\min }=$ $50 \mathrm{Myr}[16,17,70]$. The maximum time delay $t_{\max }$ is set to the Hubble time [70-77].

We assume that the binary formation rate $R_{f}\left(z_{f}\right)$ scales with the SFR. We follow the cosmic star formation model of [66] which uses the SpringerHernquist functional form [78]

$$
R_{f}(z)=\nu \frac{a e^{b\left(z-z_{m}\right)}}{a-b+b e^{\left(a\left(z-z_{m}\right)\right.}}
$$

to fit to the gamma-ray burst-based high-redshift SFR data of [79] but normalized based on the procedure described in [80,81]. This fit results in $\nu=0.146 M_{\odot} / \mathrm{yr} / \mathrm{Mpc}^{3}, z_{m}=1.72, a=2.80$, and $b=2.46$ [66]. The value of $R_{m}(z=0)$ is chosen as the local merger rate estimate from the LIGO-Virgo observations. For the rate of BBH mergers, we use the most recent published result associated with the power-law mass distribution $56_{-27}^{+44} \mathrm{Gpc}^{-3} \mathrm{yr}^{-1}$ [6]. For the BNS case, we set $R_{m}(z=0)$ to $920_{-790}^{+2220} \mathrm{Gpc}^{-3} \mathrm{yr}^{-1}$ also from [6]. Massive black holes are formed preferentially in low-metallicity environments $[16,82]$. For systems where at least one black hole has a mass larger than $30 M_{\odot}$, we reweight the star formation rate $R_{f}(z)$ by the fraction of stars with metallicities less than half the solar metallicity [17]. Following [16,17], we use the mean metallicity-redshift relation of [83] and scale it upward by a factor of 3 to account for local observations $[66,84]$.

(3) The location on the sky, the cosine of the inclination angle, the polarization, and the coalescence phase are drawn from uniform distributions.

\section{A. Detector network}

We consider two networks of third generation detectors: one with three total detectors, out of which two have the sensitivity of CE located at LIGO Hanford and LIGO Livingston locations and one with the sensitivity of ET located at the location of Virgo; and a five-detector network with one detector with the sensitivity of ET at the location of Virgo, and detectors with CE sensitivity at locations of LIGO Hanford, LIGO Livingston, LIGO India, and KAGRA. We choose these configurations for the detector networks because it was shown in [48] that the astrophysical "confusion" background from unresolved $\mathrm{BBH}$ sources is decreased by orders of magnitude, reaching $\Omega_{\mathrm{GW}}(10 \mathrm{~Hz})=$ $10^{-14}-10^{-13}$ and $\Omega_{\mathrm{GW}}(10 \mathrm{~Hz})=10^{-16}-10^{-14}$, respectively.

\section{SIMULATIONS}

We simulate a population of BBH and BNS mergers according to the procedure described in Sec. IV for a year of data. There are 76,107 BBH and 1,438,835 BNS signals in our simulation. For each source, we calculate the expected network SNR assuming perfect template match, given by

$$
\rho_{i}^{\text {net }}=\sqrt{\sum_{\operatorname{det}}\left(\rho_{i}^{\mathrm{det}}\right)^{2}},
$$

where index $i$ runs over all the sources, and $\rho_{i}^{\mathrm{det}}$,

$$
\left(\rho_{i}^{\mathrm{det}}\right)^{2}=4 \int_{0}^{\infty} \mathrm{df} \frac{\left|\tilde{h}_{i}^{\operatorname{det}}(f)\right|^{2}}{S_{h}^{\operatorname{det}}(f)},
$$

is the SNR for each source and detector pair ( $i$, det), and $\tilde{h}_{i}^{\operatorname{det}}(f)=F_{+}^{\operatorname{det}} \tilde{h}_{i,+}+F_{\times}^{\operatorname{det}} \tilde{h}_{i, \times}$ is the Fourier domain waveform projected on the detector.

We considered a source as resolvable and a part of the "foreground," whenever $\rho_{i}^{\text {net }} \geq \rho_{\text {thresh }}=12.0$. We use the 0 order PN approximation for waveforms, since the results from that and a full inspiral-merger-ringdown model agree to a great extent below $100 \mathrm{~Hz}$. It has been shown for various detector combinations that frequencies below $100 \mathrm{~Hz}$ account for more than $99 \%$ of the SNR for the stochastic search $[64,69]$. Therefore, for calculating $\Omega_{\text {error }}$, we only consider the 0th-PN model to compute the Fisher matrix for each source in our simulation.

We calculate the Fisher matrices (and the variancecovariance matrices) for all the sources in our simulation and recover a set of parameters in order to calculate $\Omega_{\text {residual,BNS }}$ and $\Omega_{\text {residual,BBH}}$.

Our results are shown plotted in Fig. 1. For the threedetector case, we find that $49 \%$ of the BNS sources are unresolved (with a network SNR $<12$ ), whereas only $1.3 \%$ of the BBH sources are unresolved. For the fivedetector case, we find that $25 \%$ of the BNS sources are unresolved while only $0.075 \%$ of the BBH sources remain unresolved. We show the results for network SNR threshold of 12 in the first two rows of Fig. 1. The first row shows the results for BBH (left: for a three-detector $3 \mathrm{G}$ network, right: for a five-detector $3 \mathrm{G}$ network) and the second row shows the results for the BNS (left: for a three-detector $3 \mathrm{G}$ network, right: for a five-detector $3 \mathrm{G}$ network). We can 


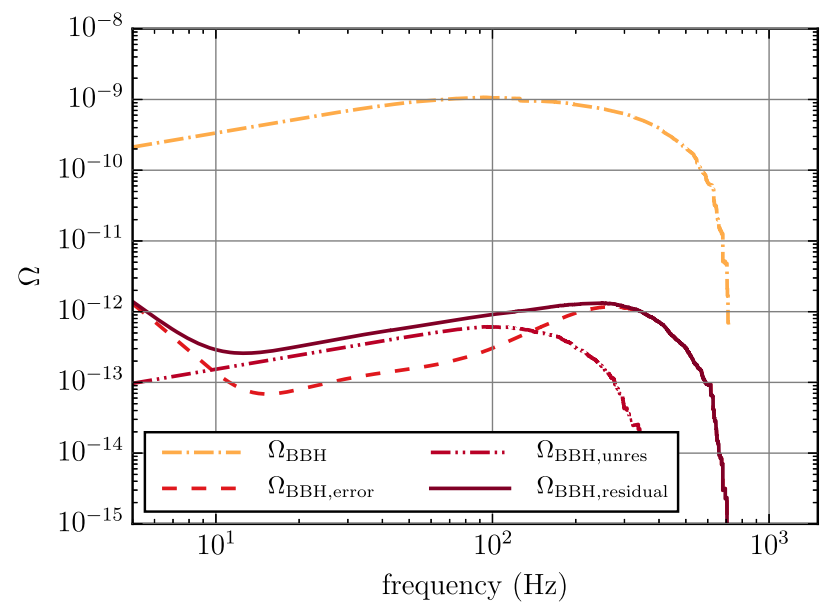

(a) BBH, HLV network

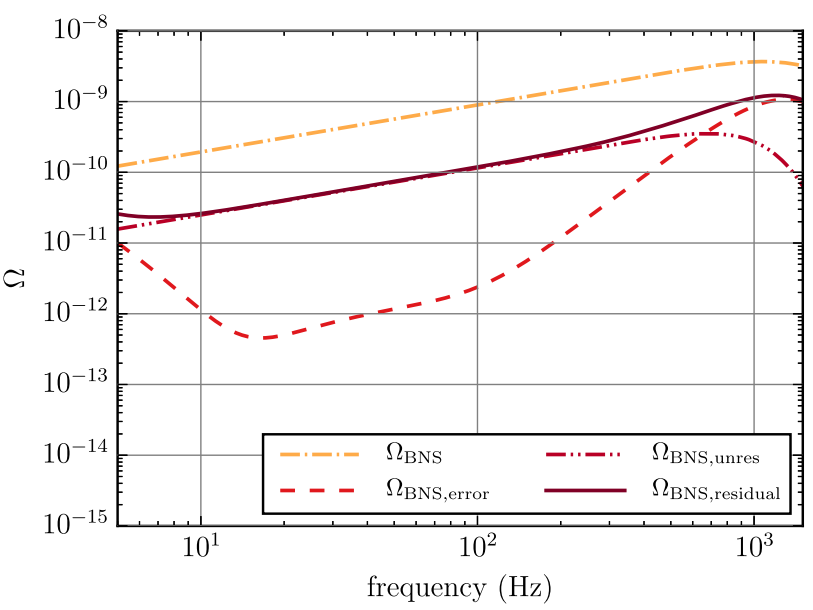

(c) BNS, HLV network

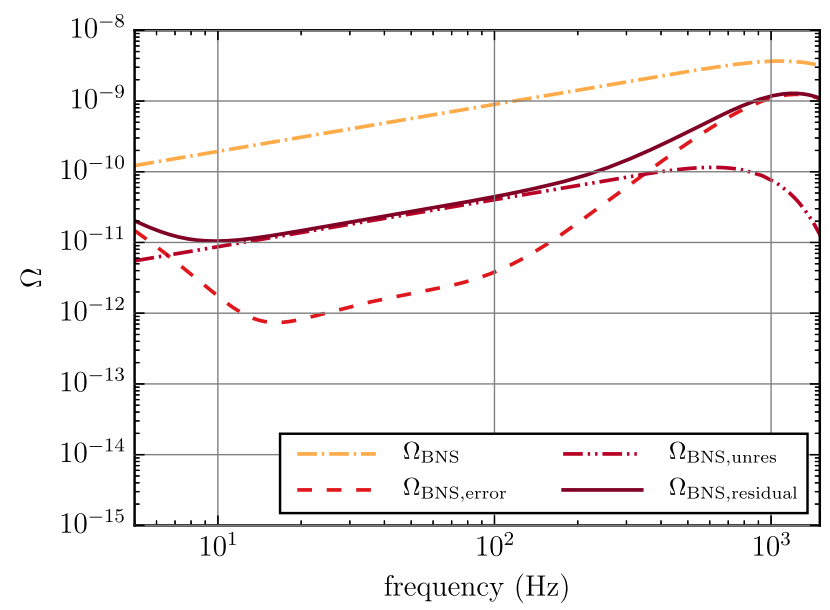

(e) BNS, HLV network, SNR threshold 8.0

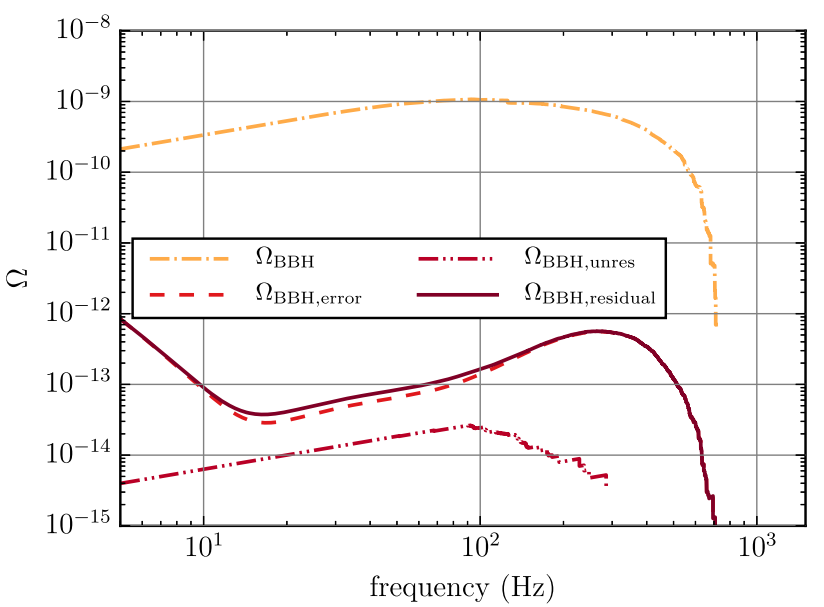

(b) BBH, HLVIK network

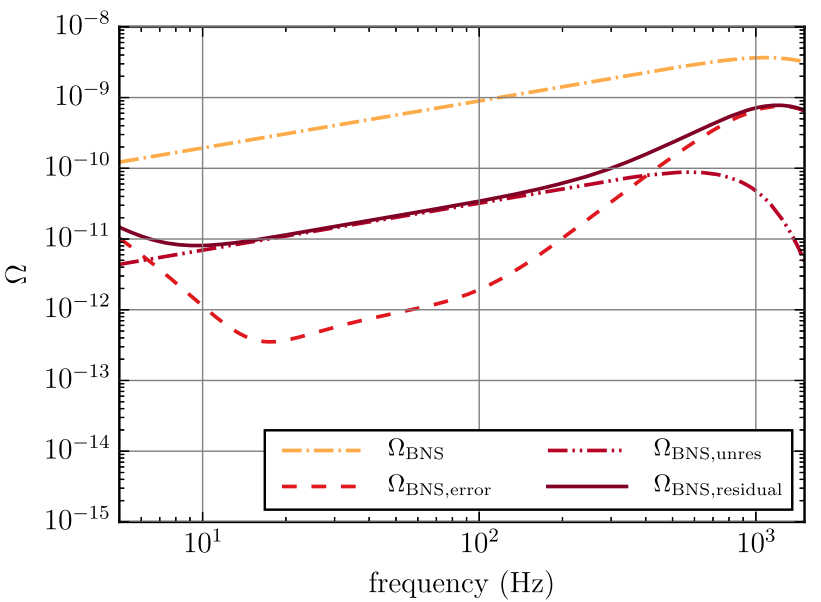

(d) BNS, HLVIK network

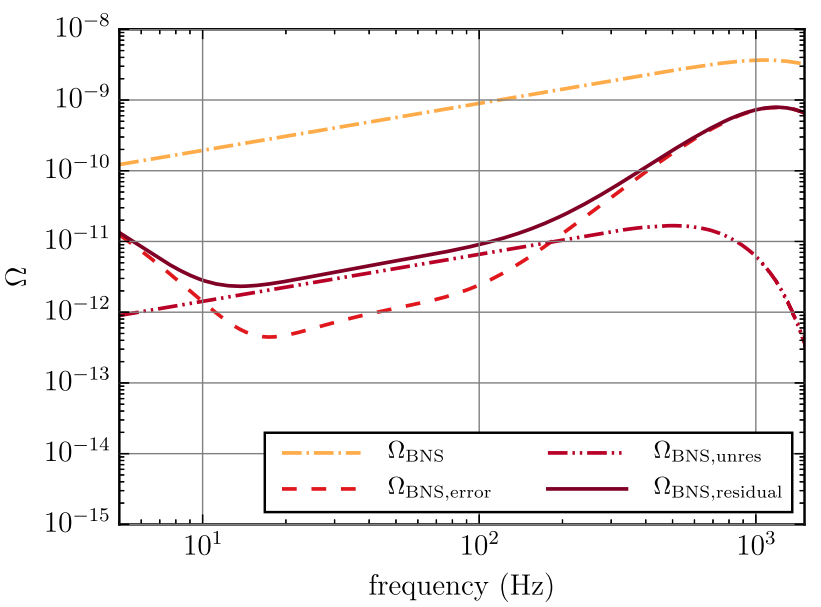

(f) BNS, HLVIK network, SNR threshold 8.0

FIG. 1. The confusion background created by the astrophysical population of merging binary black holes (top two panels) and binary neutron stars (bottom four panels) is shown plotted (dot-dashed, orange lines) together with the background from unresolved sources (dot-dot-dashed, red lines), the background that remains after imperfect subtraction of resolved sources (dashed, red lines) and the sum of the latter two (solid, deep-red lines). The left panels are for a network of three $3 \mathrm{G}$ detectors, and the right panels are for a network of five $3 \mathrm{G}$ detectors. We deem a source is resolved if the signal-to-noise it produces is $\geq 12$ for the top four panels and $\geq 8$ for the bottom two panels. 
see that the $\Omega_{\text {residual }}=\Omega_{\text {error }}+\Omega_{\text {cbc, unres }}$ depends on the network SNR threshold. The higher the network SNR threshold, the lower the $\Omega_{\text {error }}$ but higher the $\Omega_{\mathrm{cbc} \text {, unres }}$. Thus, the network SNR threshold can be varied to minimize the $\Omega_{\text {residual }}$.

For the BBH case, we have not tried to optimize the $\Omega_{\text {residual,BBH}}$, since it lies much below the $\Omega_{\text {residual,BNS }}$. For the BNS case, we can see from the second row of Fig. 1 that we may be able to lower the residual background by decreasing the network SNR threshold, since the residual is dominated by the unresolved sources. We decided to lower the network SNR threshold to 8 (the threshold at which we should be able to resolve signals in case of Gaussian noise); these results are shown in the last row of Fig. 1. With a network SNR threshold of 8, the number of unresolved BNS sources for a three (and five) network of $3 \mathrm{G}$ detectors reduces to $25 \%$ from $49 \%$ ( $7.7 \%$ from $25 \%$ ). We have managed to lower the BNS residual background by lowering the detector network SNR threshold. The residual background from the BNS sources still dominates over the $\mathrm{BBH}$ background and is the limiting factor for the primordial backgrounds we can observe. An alternative would be to follow the noise projection method described in Ref. [51], which does not require the SNR optimization procedure described here.

\section{DISCUSSION}

Conclusions of our study are summarized in Fig. 2. The figure plots the energy density in gravitational waves $\Omega(f)$ from axion inflation [85], a network of cosmic strings [24-27], a background produced during postinflation by oscillations of a fluid with an equation-of-state stiffer than radiation [86], and from postinflation preheating scenarios [87,88] aided by parametric resonance [23,89]. For reference, we show the strength of the stochastic background from vacuum fluctuations during standard inflation [18-20], although this will not be detectable by any of the foreseen ground-based detector networks; others are examples of primordial backgrounds that could be potentially detected by $3 \mathrm{G}$ detectors. The strength of the background in these examples depends on model parameters and it could be lower or higher than what is shown on the plot.

The figure also shows the sensitivity of a network of three (and five) 3G detectors to stochastic backgrounds assuming a 1-year integration but in the absence of confusion backgrounds from compact binaries or other astrophysical populations. $\Omega_{\text {error }}$ curves shown in Fig. 1 have a minima at around $15 \mathrm{~Hz}$ which follows from the sensitivity of the detector network. It is immediately apparent from Fig. 2 that the residual background, after (imperfect) subtraction of the foreground sources, from binary neutron stars will limit the strength of primordial backgrounds that could be detected by $3 \mathrm{G}$ detectors. With a network of three (and five) $3 \mathrm{G}$ detectors, the sensitivity will

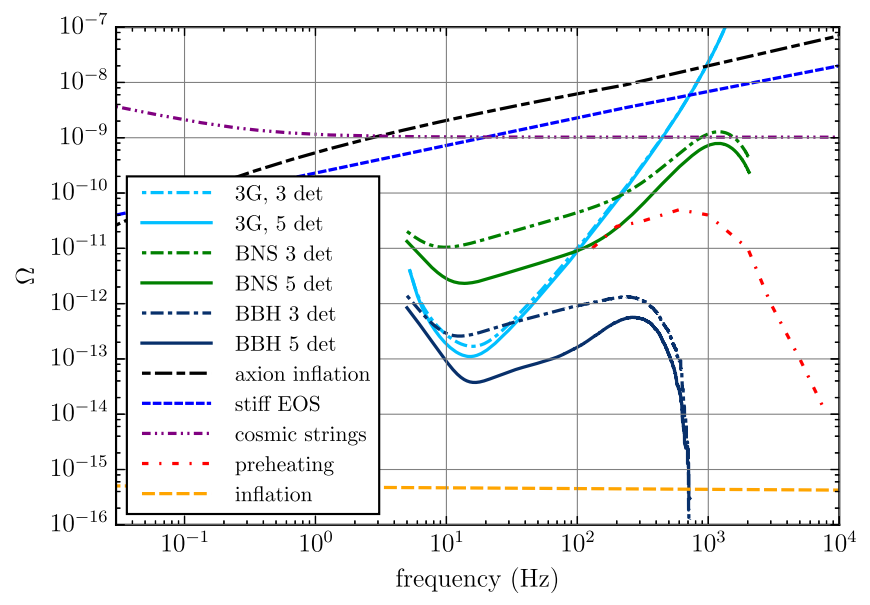

FIG. 2. Residual backgrounds after subtraction of the resolved foreground are plotted in solid (and dot-dashed) lines for a network of five $3 \mathrm{G}$ detectors (three $3 \mathrm{G}$ detectors, respectively) for the BNS cosmological population in green and $\mathrm{BBH}$ population in deep blue. Also shown are the raw sensitivity curves for a stochastic background after 1 year of integration for a network of five $3 \mathrm{G}$ detectors (solid, cyan curves) and three $3 \mathrm{G}$ detectors (dot-dashed, cyan curves) and the expected background from stiff equation-ofstate, cosmic (super) strings, preheating, and inflation.

be limited to $\Omega_{\mathrm{GW}} \geq 10^{-11}$ at $10 \mathrm{~Hz}$ (respectively, $\Omega_{\mathrm{GW}} \geq$ $3 \times 10^{-12}$ at $15 \mathrm{~Hz}$ ). The binary black hole population, on the other hand, can be fully resolved and the residual from that population has negligible effect on the raw sensitivity to stochastic backgrounds. The rate of binary neutron stars could be larger or smaller than the median rate of $R_{m}(z=0)=920_{-790}^{+2220} \mathrm{Gpc}^{-3} \mathrm{yr}^{-1}$ assumed in this paper, which would correspondingly increase or decrease the confusion background of these sources. Finally, increasing the number of $3 \mathrm{G}$ detectors from three to five improves the sensitivity to stochastic backgrounds by about factor of 5 . This is accounted by the ability of the five-detector network to detect and subtract a greater number of sources; the volume reach for a five-detector network increases by a factor $(5 / 3)^{3} \sim 4.6$ relative to a three-detector network.

Keeping in mind that the strengths of the primordial backgrounds depend on the specific model parameters that are not known, and the residual background could vary based on the uncertainty in rate of compact binary mergers and the their mass distribution, among other things, the figure shows the most promising primordial background sources that this subtraction scheme could reveal: cosmic strings, background from fluids with stiff EOS, and axion inflation.

\section{ACKNOWLEDGMENTS}

We thank Thomas Callister, Duncan Meacher, and Alan Weinstein for helpful discussions and comments. We thank Joe Romano for carefully reading the paper and providing useful comments. We thank Andrew Matas for providing useful data regarding some of the backgrounds considered 
in this paper. S. S. acknowledges the support of the Eberly Research Funds of Penn State, The Pennsylvania State University, University Park, Pennsylvania. S. S. also acknowledges the support of Chateaubriand Fellowship which led to the collaboration between S. S. and T. R. and the start of this project. B.S.S. was supported in part by
NSF Grants No. PHY-1836779, No. AST-1716394, and No. AST-1708146 and the Science and Technology Facilities Council of the United Kingdom. We acknowledge the use of ICDS cluster at Penn State for the simulations in this work. This paper has the LIGO document number LIGO-P2000009.
[1] B. P. Abbott, R. Abbott, T. D. Abbott, M. R. Abernathy, F. Acernese, K. Ackley, C. Adams, T. Adams, P. Addesso, R. X. Adhikari et al., Phys. Rev. Lett. 116, 061102 (2016).

[2] B. P. Abbott, R. Abbott, T. D. Abbott, M. R. Abernathy, F. Acernese, K. Ackley, C. Adams, T. Adams, P. Addesso, R. X. Adhikari et al., Phys. Rev. Lett. 116, 241103 (2016).

[3] B. P. Abbott, R. Abbott, T. D. Abbott, F. Acernese, K. Ackley, C. Adams, T. Adams, P. Addesso, R. X. Adhikari et al., Phys. Rev. Lett. 118, 221101 (2017).

[4] B. P. Abbott, R. Abbott, T. D. Abbott, F. Acernese, K. Ackley, C. Adams, T. Adams, P. Addesso, R. X. Adhikari et al., Astrophys. J. Lett. 851, L35 (2017).

[5] B. P. Abbott, R. Abbott, T. D. Abbott, F. Acernese, K. Ackley, C. Adams, T. Adams, P. Addesso, R. X. Adhikari et al., Phys. Rev. Lett. 119, 141101 (2017).

[6] B. P. Abbott et al. (LIGO Scientific, Virgo Collaborations), Phys. Rev. X 9, 031040 (2019).

[7] B. P. Abbott, R. Abbott, T. D. Abbott, F. Acernese, K. Ackley, C. Adams, T. Adams, P. Addesso, R. X. Adhikari et al. (LIGO Scientific Collaboration and Virgo Collaboration), Phys. Rev. Lett. 119, 161101 (2017).

[8] B. P. Abbott et al. (LIGO Scientific, Virgo Collaborations), Astrophys. J. Lett. 892, L3 (2020).

[9] X. J. Zhu, E. Howell, T. Regimbau, D. Blair, and Z. H. Zhu, Astrophys. J. 739, 86 (2011).

[10] P. A. Rosado, Phys. Rev. D 84, 084004 (2011).

[11] S. Marassi, R. Schneider, G. Corvino, V. Ferrari, and S. P. Zwart, Phys. Rev. D 84, 124037 (2011).

[12] C. Wu, V. Mandic, and T. Regimbau, Phys. Rev. D 85, 104024 (2012).

[13] X.-J. Zhu, E. J. Howell, D. G. Blair, and Z.-H. Zhu, Mon. Not. R. Astron. Soc. 431, 882 (2013).

[14] J. Aasi, B. P. Abbott, R. Abbott, T. Abbott, M. R. Abernathy, K. Ackley, C. Adams, T. Adams, P. Addesso, R. X. Adhikari et al., Classical Quantum Gravity 32, 074001 (2015).

[15] The Virgo Collaboration, Classical Quantum Gravity 32, 024001 (2015).

[16] B. P. Abbott, R. Abbott, T. D. Abbott, M. R. Abernathy, F. Acernese, K. Ackley, C. Adams, T. Adams, P. Addesso, R. X. Adhikari et al. (LIGO Scientific Collaboration and Virgo Collaboration), Phys. Rev. Lett. 116, 131102 (2016).

[17] B. P. Abbott, R. Abbott, T. D. Abbott, F. Acernese, K. Ackley, C. Adams, T. Adams, P. Addesso, R. X. Adhikari et al. (LIGO Scientific Collaboration and Virgo Collaboration), Phys. Rev. Lett. 120, 091101 (2018).

[18] L. P. Grishchuk, Sov. Phys. JETP 40, 409 (1975), https://ui .adsabs.harvard.edu/abs/1975JETP...40..409G/abstract.
[19] A. A. Starobinskiŭ, JETP Lett. 30, 682 (1979), https://ui .adsabs.harvard.edu/abs/1979ZhPmR..30..719S/abstract.

[20] L. P. Grishchuk, Phys. Rev. D 48, 3513 (1993).

[21] M. Gasperini and G. Veneziano, Astropart. Phys. 1, 317 (1993).

[22] A. Buonanno, M. Maggiore, and C. Ungarelli, Phys. Rev. D 55, 3330 (1997).

[23] J. F. Dufaux, D. G. Figueroa, and J. García-Bellido, Phys. Rev. D 82, 083518 (2010).

[24] T. Damour and A. Vilenkin, Phys. Rev. D 71, 063510 (2005).

[25] X. Siemens, V. Mandic, and J. Creighton, Phys. Rev. Lett. 98, 111101 (2007).

[26] S. Ölmez, V. Mandic, and X. Siemens, Phys. Rev. D 81, 104028 (2010).

[27] T. Regimbau, S. Giampanis, X. Siemens, and V. Mandic, Phys. Rev. D 85, 066001 (2012).

[28] C. Caprini, R. Durrer, and G. Servant, Phys. Rev. D 77, 124015 (2008).

[29] C. Caprini, R. Durrer, T. Konstandin, and G. Servant, Phys. Rev. D 79, 083519 (2009).

[30] C. Caprini, R. Durrer, and G. Servant, J. Cosmol. Astropart. Phys. 12 (2009) 024.

[31] M. Maggiore, Phys. Rep. 331, 283 (2000).

[32] P. Binétruy, A. Bohé, C. Caprini, and J.-F. Dufaux, J. Cosmol. Astropart. Phys. 06 (2012) 27.

[33] T. Regimbau, Res. Astron. Astrophys. 11, 369 (2011).

[34] A. Buonanno, G. Sigl, G. G. Raffelt, H. T. Janka, and E. Müller, Phys. Rev. D 72, 084001 (2005).

[35] P. Sandick, K. A. Olive, F. Daigne, and E. Vangioni, Phys. Rev. D 73, 104024 (2006).

[36] S. Marassi, R. Schneider, and V. Ferrari, Mon. Not. R. Astron. Soc. 398, 293 (2009).

[37] X. J. Zhu, E. Howell, and D. Blair, Mon. Not. R. Astron. Soc. 409, L132 (2010).

[38] T. Regimbau and J.A. de Freitas Pacheco, Astron. Astrophys. 376, 381 (2001).

[39] P. A. Rosado, Phys. Rev. D 86, 104007 (2012).

[40] T. Regimbau and J.A. de Freitas Pacheco, Astron. Astrophys. 447, 1 (2006).

[41] E. Howell, T. Regimbau, A. Corsi, D. Coward, and R. Burman, Mon. Not. R. Astron. Soc. 410, 2123 (2011).

[42] S. Marassi, R. Ciolfi, R. Schneider, L. Stella, and V. Ferrari, Mon. Not. R. Astron. Soc. 411, 2549 (2011).

[43] C. J. Wu, V. Mandic, and T. Regimbau, Phys. Rev. D 87, 042002 (2013).

[44] J. C. N. de Araujo and G. F. Marranghello, Gen. Relativ. Gravit. 41, 1389 (2009). 
[45] V. Ferrari, S. Matarrese, and R. Schneider, Mon. Not. R. Astron. Soc. 303, 258 (1999).

[46] E. Howell, D. Coward, R. Burman, D. Blair, and J. Gilmore, Mon. Not. R. Astron. Soc. 351, 1237 (2004).

[47] X. J. Zhu, X. L. Fan, and Z. H. Zhu, Astrophys. J. 729, 59 (2011).

[48] T. Regimbau, M. Evans, N. Christensen, E. Katsavounidis, B. Sathyaprakash, and S. Vitale, Phys. Rev. Lett. 118, 151105 (2017).

[49] M. Punturo, M. Abernathy, F. Acernese, B. Allen, N. Andersson, K. Arun, F. Barone, B. Barr, M. Barsuglia, M. Beker et al., Classical Quantum Gravity 27, 194002 (2010).

[50] B. P. Abbott, R. Abbott, T. D. Abbott, M. R. Abernathy, K. Ackley, C. Adams, P. Addesso, R. X. Adhikari et al., Classical Quantum Gravity 34, 044001 (2017).

[51] J. Harms, C. Mahrdt, M. Otto, and M. Priess, Phys. Rev. D 77, 123010 (2008).

[52] J. Crowder and N. J. Cornish, Phys. Rev. D 72, 083005 (2005).

[53] C. Cutler and J. Harms, Phys. Rev. D 73, 042001 (2006).

[54] B. S. Sathyaprakash and S. V. Dhurandhar, Phys. Rev. D 44, 3819 (1991).

[55] B.F. Schutz, in Gravitational Wave Data Analysis (Springer, Netherlands, 1989).

[56] B. Allen and J. D. Romano, Phys. Rev. D 59, 102001 (1999).

[57] B. Allen and J. D. Romano, Phys. Rev. D 59, 102001 (1999).

[58] J. Aasi et al. (LIGO Scientific, VIRGO Collaborations), Phys. Rev. D 91, 022003 (2015).

[59] M. W. Coughlin, A. Cirone, P. Meyers, S. Atsuta, V. Boschi, A. Chincarini, N. L. Christensen, R. De Rosa, A. Effler, I. Fiori, M. Gołkowski, M. Guidry, J. Harms, K. Hayama, Y. Kataoka, J. Kubisz, A. Kulak, M. Laxen, A. Matas, J. Mlynarczyk et al., Phys. Rev. D 97, 102007 (2018).

[60] Planck Collaboration, Astron. Astrophys. 594, A13 (2016).

[61] K. G. Arun, B. R. Iyer, B. S. Sathyaprakash, and P. A. Sundararajan, Phys. Rev. D 71, 084008 (2005).

[62] A. Buonanno, B. R. Iyer, E. Ochsner, Y. Pan, and B. S. Sathyaprakash, Phys. Rev. D 80, 084043 (2009).

[63] T. Regimbau, T. Dent, W. Del Pozzo, S. Giampanis, T. G. F. Li, C. Robinson, C. Van Den Broeck, D. Meacher, C. Rodriguez, B. S. Sathyaprakash, and K. Wójcik, Phys. Rev. D 86, 122001 (2012).

[64] T. Regimbau, D. Meacher, and M. Coughlin, Phys. Rev. D 89, 084046 (2014).

[65] D. Meacher, M. Coughlin, S. Morris, T. Regimbau, N. Christensen, S. Kandhasamy, V. Mandic, J. D. Romano, and E. Thrane, Phys. Rev. D 92, 063002 (2015).

[66] E. Vangioni, K. A. Olive, T. Prestegard, J. Silk, P. Petitjean, and V. Mandic, Mon. Not. R. Astron. Soc. 447, 2575 (2015).
[67] E. E. Salpeter, Astrophys. J. 121, 161 (1955).

[68] B. P. Abbott, R. Abbott, T. D. Abbott, M. R. Abernathy, F. Acernese, K. Ackley, C. Adams, T. Adams, P. Addesso, R. X. Adhikari et al. (LIGO Scientific Collaboration and Virgo Collaboration), Phys. Rev. X 6, 041015 (2016).

[69] D. Meacher, M. Coughlin, S. Morris, T. Regimbau, N. Christensen, S. Kandhasamy, V. Mandic, J. D. Romano, and E. Thrane, Phys. Rev. D 92, 063002 (2015).

[70] M. Dominik, K. Belczynski, C. Fryer, D. E. Holz, E. Berti, T. Bulik, I. Mandel, and R. O'Shaughnessy, Astrophys. J. 779, 72 (2013).

[71] K. Belczynski, V. Kalogera, and T. Bulik, Astrophys. J. 572, 407 (2002).

[72] S. Ando, J. Cosmol. Astropart. Phys. 04 (2004) 007.

[73] K. Belczynski, R. Perna, T. Bulik, V. Kalogera, N. Ivanova, and D. Q. Lamb, Astrophys. J. 648, 1110 (2006).

[74] E. Berger, D. B. Fox, P. A. Price, E. Nakar, A. Gal-Yam, D. E. Holz, B. P. Schmidt, A. Cucchiara, S. B. Cenko, S. R. Kulkarni, A. M. Soderberg, D. A. Frail, B. E. Penprase, A. Rau, E. Ofek et al., Astrophys. J. 664, 1000 (2007).

[75] E. Nakar, Phys. Rep. 442, 166 (2007); the Hans Bethe Centennial Volume 1906-2006.

[76] R. O'Shaughnessy, K. Belczynski, and V. Kalogera, Astrophys. J. 675, 566 (2008).

[77] M. Dominik, K. Belczynski, C. Fryer, D. E. Holz, E. Berti, T. Bulik, I. Mandel, and R. O'Shaughnessy, Astrophys. J. 759, 52 (2012).

[78] L. Hernquist and V. Springel, Mon. Not. R. Astron. Soc. 341, 1253 (2003).

[79] M. D. Kistler, H. Yuksel, and A. M. Hopkins, arXiv:1305 .1630 .

[80] M. Trenti, R. Perna, and S. Tacchella, Astrophys. J. Lett. 773, L22 (2013).

[81] P. S. Behroozi and J. Silk, Astrophys. J. 799, 32 (2015).

[82] B. P. Abbott et al. (LIGO Scientific, Virgo Collaborations), Astrophys. J. 818, L22 (2016).

[83] P. Madau and M. Dickinson, Annu. Rev. Astron. Astrophys. 52, 415 (2014).

[84] K. Belczynski, D. E. Holz, T. Bulik, and R. O'Shaughnessy, Nature (London) 534, 512 (2016).

[85] N. Barnaby, E. Pajer, and M. Peloso, Phys. Rev. D 85, 023525 (2012).

[86] L. A. Boyle and A. Buonanno, Phys. Rev. D 78, 043531 (2008).

[87] S. Y. Khlebnikov and I. I. Tkachev, Phys. Rev. D 56, 653 (1997).

[88] D. Tilley and R. Maartens, Classical Quantum Gravity 17, 2875 (2000).

[89] D. G. Figueroa and F. Torrenti, J. Cosmol. Astropart. Phys. 10 (2017) 057. 